\title{
Design and modeling of an additive manufactured thin shell for x-ray astronomy
}

\author{
Charlotte Feldman, Carolyn Atkins, David Brooks, \\ Stephen Watson, William Cochrane, Melanie Roulet, \\ Richard Willingale \& Peter Doel
}

\section{Published version information:}

Citation: $\mathrm{CH}$ Feldman et al. "Design and modeling of an additive manufactured thin shell for x-ray astronomy." Proc SPIE 10399 (2017): 103991H. Is in proceedings of: Optics for EUV, X-Ray, and Gamma-Ray Astronomy VIII, San Diego, California, USA, 6-10 Aug 2017.

doi: $\underline{10.1117 / 12.2273422}$

This version is made available in accordance with publisher policies. Please cite only the published version using the reference above. 


\section{Design and modeling of an additive manufactured thin shell for $\mathrm{x}$-ray astronomy}

Charlotte Feldman, Carolyn Atkins, David Brooks, Stephen Watson, William Cochrane, et al. 


\title{
Design and modelling of an additive manufactured thin shell for X-ray astronomy
}

\author{
Charlotte Feldman ${ }^{\mathrm{a}}$, Carolyn Atkins ${ }^{\mathrm{b}}$, David Brooks ${ }^{\mathrm{c}}$, Stephen Watson ${ }^{\mathrm{b}}$, William Cochrane ${ }^{\mathrm{b}}$, \\ Melanie Roulet ${ }^{\mathrm{d}, \mathrm{b}}$, Richard Willingale ${ }^{\mathrm{a}}$, and Peter Doel ${ }^{\mathrm{c}}$ \\ ${ }^{a}$ University of Leicester, University Road, Leicester, LE1 7RH, UK \\ ${ }^{\mathrm{b}}$ UK Astronomy Technology Centre, Royal Observatory, Blackford Hill View, Edinburgh, \\ EH9 3HJ, UK \\ 'University College London, Department of Physics and Astronomy, Gower Street, London, \\ WC1E 6BT, UK \\ dAix Marseille Univ, CNRS, Laboratoire d'Astrophysique de Marseille, Marseille, France
}

\begin{abstract}
Future X-ray astronomy missions require light-weight thin shells to provide large collecting areas within the weight limits of launch vehicles, whilst still delivering angular resolutions close to that of Chandra (0.5 arc seconds). Additive manufacturing ( $\mathrm{AM}$ ), also known as 3D printing, is a well-established technology with the ability to construct or 'print' intricate support structures, which can be both integral and light-weight, and is therefore a candidate technique for producing shells for space-based X-ray telescopes. The work described here is a feasibility study into this technology for precision X-ray optics for astronomy and has been sponsored by the UK Space Agency's National Space Technology Programme. The goal of the project is to use a series of test samples to trial different materials and processes with the aim of developing a viable path for the production of an X-ray reflecting prototype for astronomical applications. The initial design of an AM prototype X-ray shell is presented with ray-trace modelling and analysis of the X-ray performance. The polishing process may cause print-through from the light-weight support structure on to the reflecting surface. Investigations in to the effect of the print-through on the X-ray performance of the shell are also presented.
\end{abstract}

Keywords: X-ray astronomy, X-ray telescopes, Additive manufacture, Thin mirrors

\section{INTRODUCTION}

All future large scale telescope missions require huge reflecting areas (James Webb Space Telescopes (JWST) primary mirror is $6.5 \mathrm{~m}_{\text {across }}{ }^{1}$ ) which leads to a large number of components. However, to reach the science goals, these mirrors need to be very high precision with low surface roughness and a very good figure. This is true for most wavebands including infrared (JWST) and X-ray (Athena). An example is ESA's Athena mission (launch 2028) which will have a focal length of $12 \mathrm{~m}$, a $1.4 \mathrm{~m}^{2}$ area at $1 \mathrm{keV}$, weigh $5000 \mathrm{~kg}$ and a resolution of 5". In comparison NASA's current mission Chandra has a resolution of 0.5 ", a focal length of 10 $\mathrm{m}$, but only $400 \mathrm{~cm}^{2}$ area at $1 \mathrm{keV}$ and weighs $5000 \mathrm{~kg}$.

In order to be light-weight for launch, but achieve large collecting areas, a large number of high precision components are required, which need to be very thin, making production, mounting and support structures very difficult to produce. Especially as they have to withstand the high vibration and g-forces during launch.

There is therefore a requirement for all future space based telescopes and optics to be: light-weight for launch, easy and cost effective to produce, high precision to achieve science goals, have large areas which means large numbers of components, thin to achieve large areas whilst still being able to launch, and well supported to survive launch without distortion. One possible solution is to use Additive Manufacturing (AM), also known as 3D printing, to "print" an optic with inbuilt light-weight support structure, with an optical

Optics for EUV, X-Ray, and Gamma-Ray Astronomy VIII, edited by Stephen L. O'Dell, Giovanni Pareschi, Proc. of SPIE Vol. 10399, 103991H · C 2017 SPIE · CCC code: 0277-786X/17/\$18 · doi: 10.1117/12.2273422 


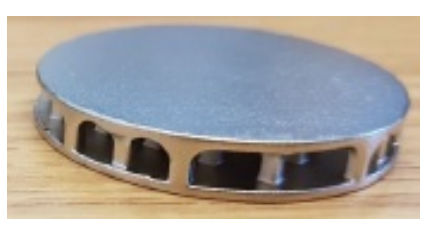

Figure 1. An AM produced aluminium sample of $4 \mathrm{~cm}$ diameter with a simple arches support structure. ${ }^{4}$

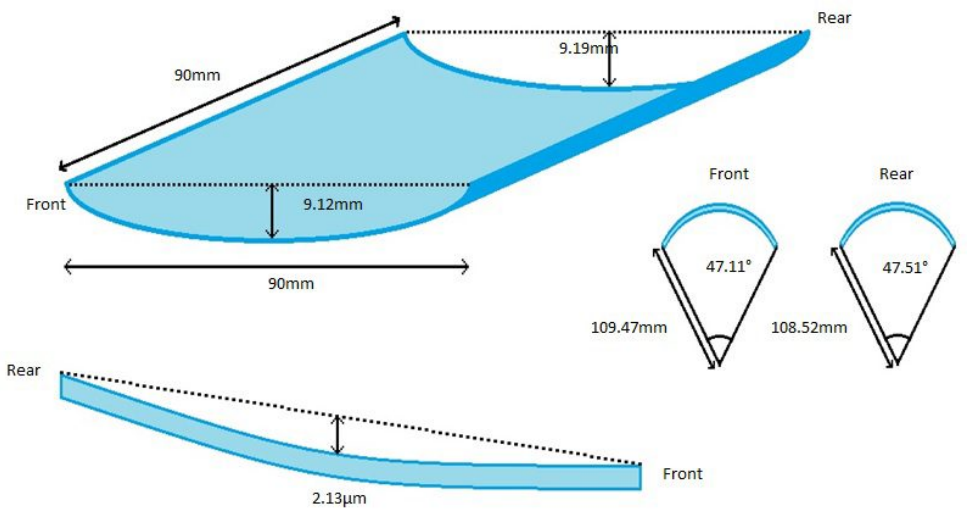

Figure 2. The AM prototype design to fit in to the TTF at the University of Leicester.

surface which could be polished to the required finish to achieve a high angular resolution. Currently AM is widely used within the aerospace ${ }^{2,3}$ industry but it is yet to be used for space-based optical components.

As described in C. Atkins et al., ${ }^{4}$ initial research, has been funded by a UK Space Agency pathfinder grant in order to investigate AM for space based optics. This is a 6 month grant which will end in September 2017 and has enabled the production of small scale samples to investigate the quality of 3D printed optical surfaces which can be polished to produce high precision reflecting surfaces. This project is looking at both the theoretical and experimental sides. The theoretical theme aims to design a high quality reflecting X-ray shell and effective light-weighting support structures. The experimental theme ${ }^{4}$ is looking at $3 \mathrm{D}$ printed samples of different materials (an example of which is shown in Figure 1) to investigate the quality of both the printed surface and how easy it is to polish and the optical surface that can be achieved.

It is hoped that by combining the results from the experimental theme ${ }^{4}$ and using Finite Element Analysis (FEA) models to optimise the light-weighted support structure, the first AM X-ray prototype shell will be produced for testing. The design of the AM prototype will be completed within the ray-tracing software and will build on the previous work from the Smart X-ray Optics (SXO) consortium. ${ }^{5-7}$ The aim is to see if it is possible to produce an X-ray optic with the resolution of Chandra but at a fraction of the weight.

\section{PROTOTYPE DESIGN}

The University of Leicester has a $27 \mathrm{~m}$ long X-ray beam line, the Tunnel Test Facility (TTF), capable of producing X-rays in the range of $0.5-100 \mathrm{keV}$, and ending in an experiment chamber with mounting for individual optics and an imaging Micro Channel Plate (MCP) detector. The detector chamber opens in to an ISO class 5 clean room to ensure a continual clean environment for the optics and equipment.

As described in C. Feldman et al., ${ }^{7}$ a support structure was developed to be able to support the optic for the SXO project within the TTF facility approximately $4 \mathrm{~m}$ from the detector. Building on this previous work and equipment, an AM prototype design for an ellipsoidal segment for simple point to point focusing to sit in the same position as the SXO optic, was designed. The SXO optic was $300 \mathrm{~mm}$ by $100 \mathrm{~mm}$ but this

Further author information: Charly Feldman:

E-mail: chf7@le.ac.uk, Telephone: +44 (0)116 2525084 


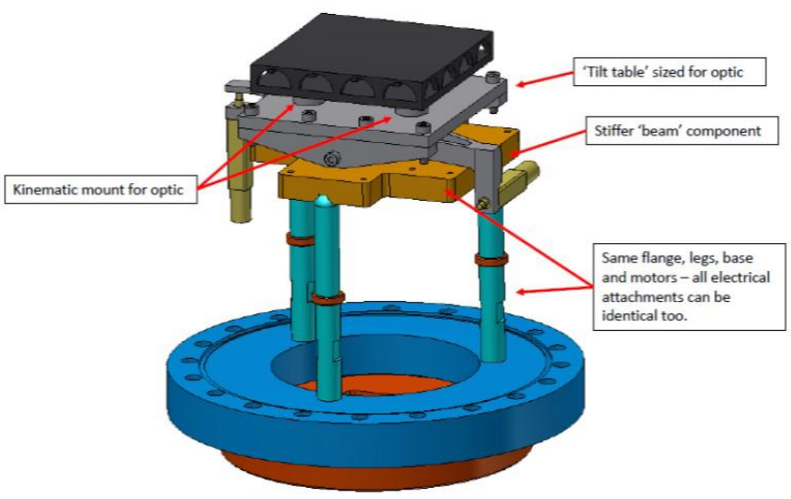

Figure 3. The redesigned mounting from the previous SXO work ${ }^{7}$ to mount the AM prototype within the TTF.
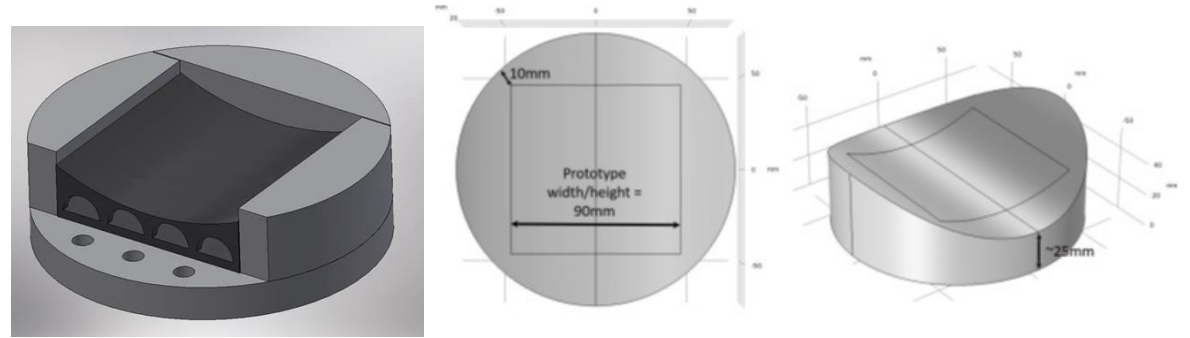

Figure 4. A CAD representation of the prototype and wasters to be printed.

AM prototype will be just $90 \mathrm{~mm}$ by $90 \mathrm{~mm}$ to ease mounting, manufacture and polishing. The details of the prototype design are given in Figure 2. A design has also been developed to re-engineer the SXO mounting to support the AM prototype within the TTF and is shown in Figure 3. The smaller prototype and redesigned mounting will allow the full assembly to be installed through the flange, which was not possible with the SXO design.

In order to polish the optical surface of the AM prototype, wasters are required around all edges, to prevent a 'turned-down' edge being created via the grinding and polishing processes. A design has been created for the wasters to be printed with the prototype but for them to be attachable and detachable after polishing. The polishing tools will also be printed at the same time as the prototype. The design for the prototype + wasters is shown in Figure 4.

\section{MODELLING}

All the modelling of the prototype has been performed in a ray-tracing program called Q, which has been designed and developed by R. Willingale at the University of Leicester. By using the sequential ray tracing code in Q, the optics behaviour as it interacts with X-rays at different wavelengths can be modelled and studied. Q enables the user to specify the size, direction and type of optic, source and the detector. The optic is specified by setting the axis, the normal, the radius of curvature, the surface type and quality, and the position of the optic. The surface quality and material can also be selected, so a gold coated nickel mirror, or an iridium coated nickel Wolter- 1 shell could be modelled. Using these values, the software can predict how the X-rays will react with the optic and therefore what results would be seen by a detector. Deformation matrices can also be imported to deform the optic surface within the ray-tracing.

\subsection{BASELINE DESIGN}

An ellipsoidal segment was chosen for the AM prototype as a simple point to point focusing test. The model was set up to simulate the TTF, with the source and detector at the foci of the ellipse and the segment being $4.4 \mathrm{~m}$ from the detector. The segment was specified to be $90 \mathrm{~mm}$ in length and $90 \mathrm{~mm}$ in width. The 


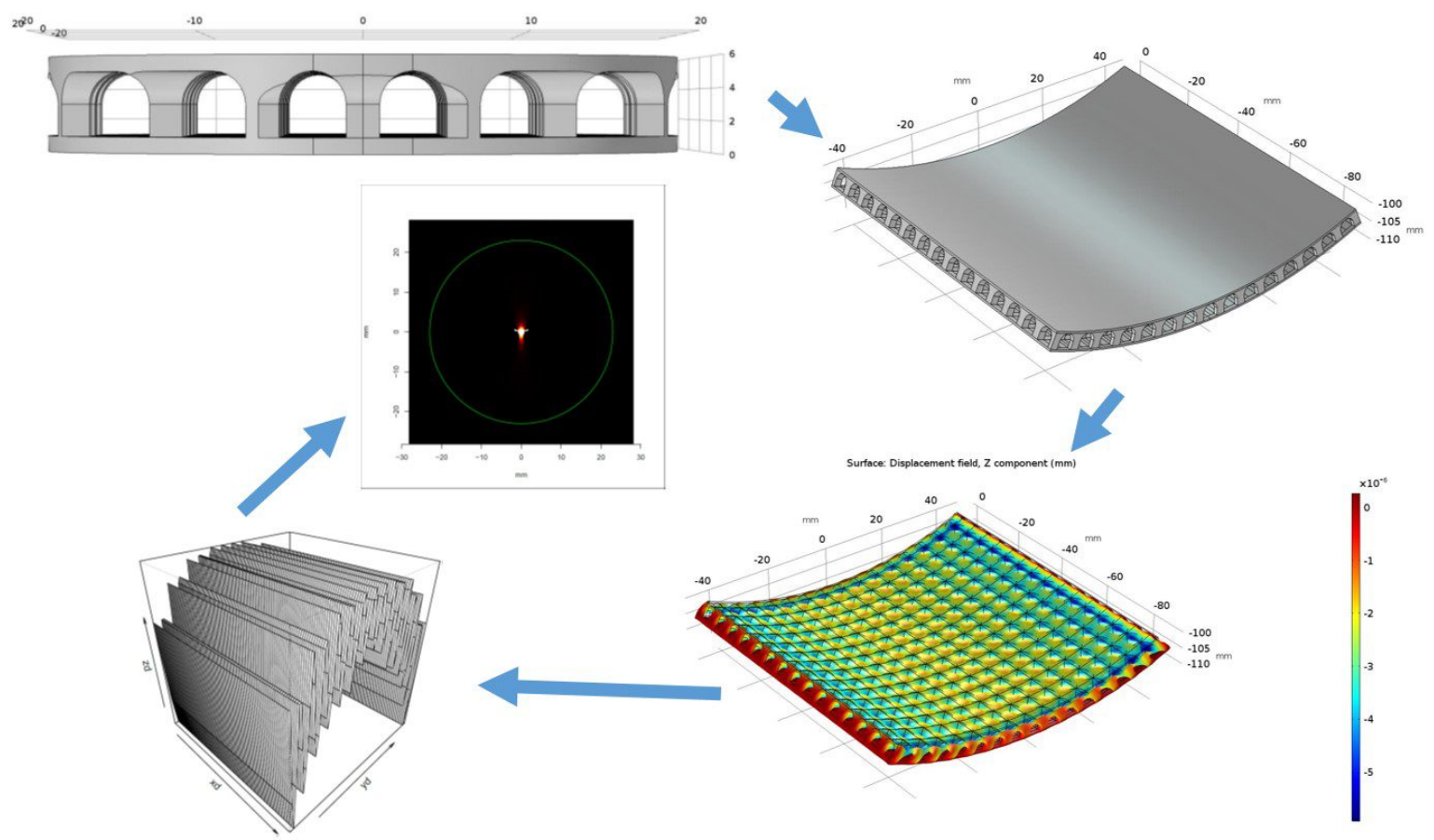

Figure 5. A graphical representation of the theory loop created to look at different light-weighted support structures.

semi-minor and semi-major axes of the ellipse were specified and the orientation of the ellipse was set. A gold surface was specified and initially a very low roughness surface was modelled ( $5 \AA \mathrm{rms})$. An expected detected image was also produced. The low roughness surface was then replaced by a rougher surface, based on the experimental data, and deformation matrices were added to describe the print-through of the light-weighted support structure on to the optical surface due to polishing.

\subsection{INCORPORATING THE FEA MODELS}

A theory loop has been created between the FEA simulations produced by C. Atkins and the ray-trace software. In the first simulations the support structures designed for the small AM samples were extended under the full prototype design within the FEA software. A load is then applied representing the polishing pressure, to create an FEA model of the suspected print-through post polishing. This surface is then exported from the FEA and imported in to the ray-tracing software as a deformation matrix and a prediction of the detected image is then produced. Using this information, alterations to the support structure can be explored and the changes in the Point Spread Function (PSF) of the optic in the detected image can be compared. This should lead to the development of a possible optimised light-weighted support structure for an AM X-ray optic. A graphical representation of this loop is demonstrated in Figure 5.

The FEA model generates the vertical or z-displacement of the optical surface and a radial displacement across the prototype surface of the print through that would be seen post polishing of a specific support structure. Examples of the images or deformation matrices produced by the software, are shown in Figure 6.

A contact profilometer was used to measure the surfaces of the samples, as described in C. Atkins et al. ${ }^{4}$ A set of these measurements was compared to an FEA simulation of the same measurement of the same surface and the results of the two are shown in Figure 7. Both data sets are shown after a direct fast Fourier transform has been applied. The FEA data demonstrates where the suspected print-though should occur and its simulated magnitude, however, the measurements made on the sample indicate that the magnitude is in fact 100x larger. For this reason, all FEA data has been scaled within the ray-trace model in order to be more realistic. 


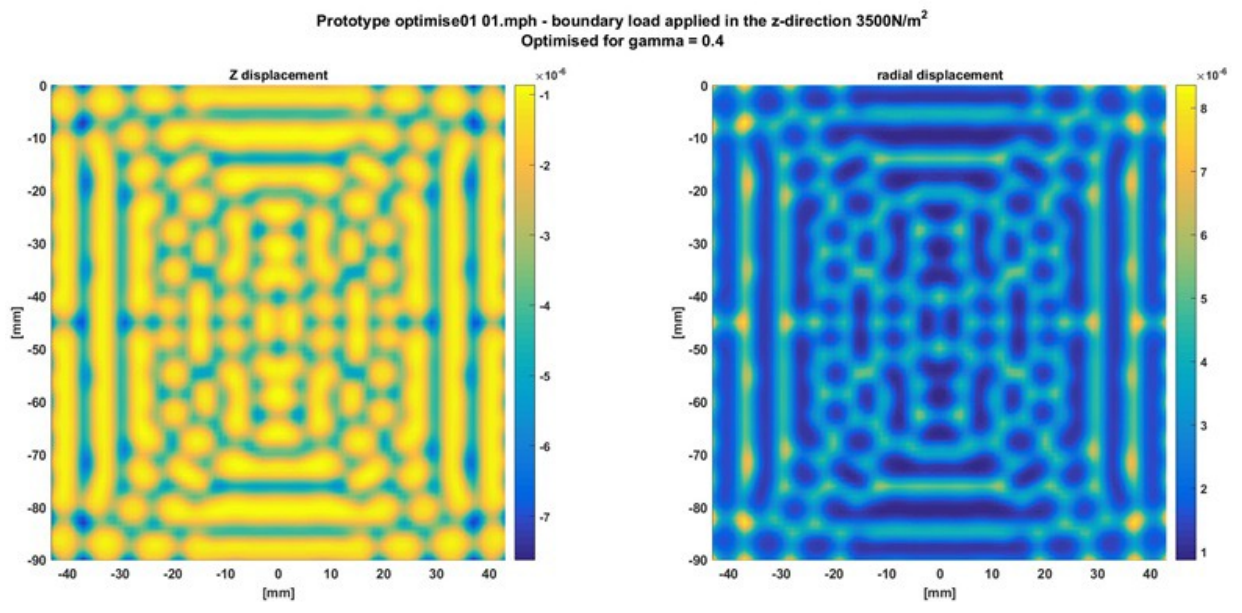

Figure 6. Examples of the images or exported data from the FEA models of the print through post polishing of an optimised support structure
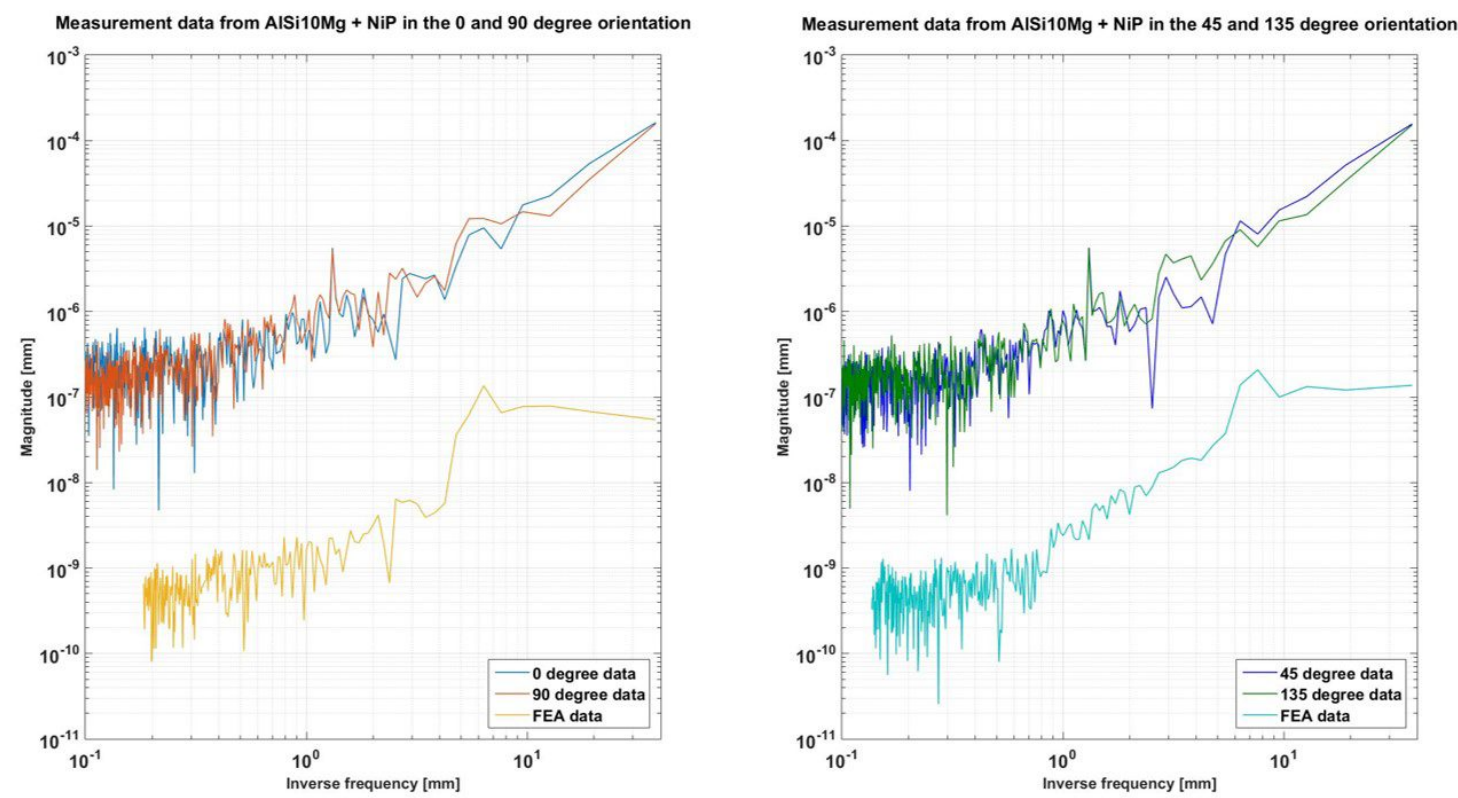

Figure 7. A comparison of fast Fourier transforms of data from a contact profilometer of one of the small samples ${ }^{4}$ obtained from both real data and an FEA simulation of the same measurement on a simulation of the same surface. 

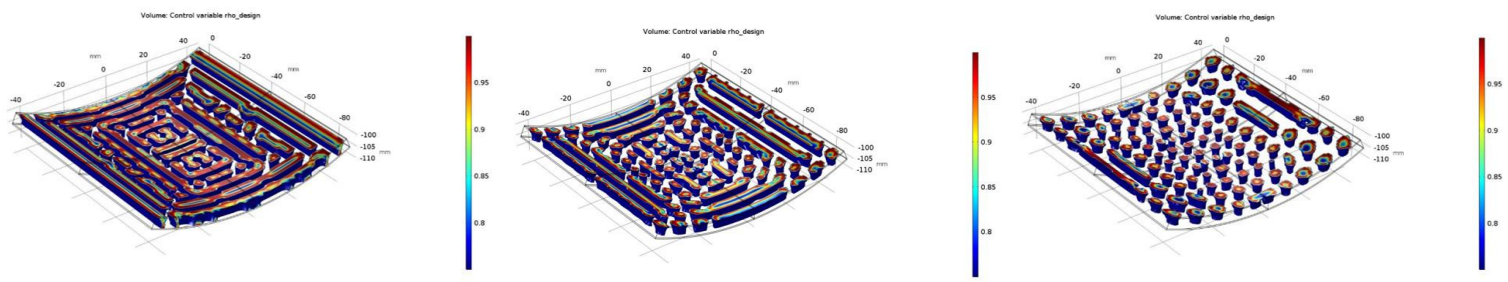

Figure 8. The first optimised support structure "Optimise 1". From left to right (Optimise 1_2, Optimise 1_1 and Optimise 1_3), $\gamma$ has been set to $0.5,0.4$ and 0.3 respectively.
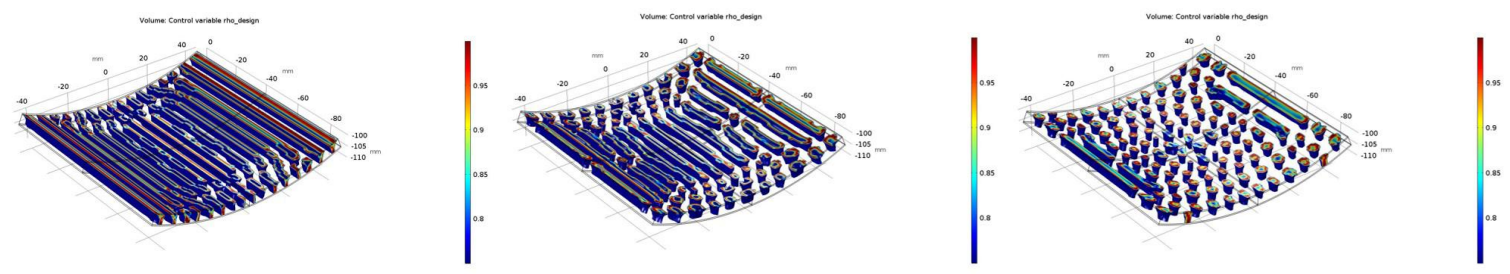

Figure 9. The second optimised support structure "Optimise 2". From left to right (Optimise 2_1, Optimise 2_2 and Optimise 2_3), $\gamma$ has been set to $0.5,0.4$ and 0.3 respectively.

\subsection{THE FIRST OPTIMISED DESIGNS}

As described in Section 4 of C. Atkins et al., ${ }^{4}$ COMSOL Multiphysics ${ }^{8}$ contains a topological optimisation tool which can be used to specify the amount of material to be removed from a specific support structure design given certain boundary conditions. This tool was used to design two possible optimised support structures. The first "Optimise 1" is shown in Figure 8 with three values for $\gamma$, the amount of material to be removed. In the left hand image, $\gamma=0.4$ which means $40 \%$ of the original material remains. In the centre and right hand images, $\gamma$ is set to 0.5 and 0.3 respectively. As can be seen, at $30 \%$ material remaining, the structure breaks down and a series of posts remain. The second is "Optimise 2" and is shown in Figure 9 where $\gamma$ has been set to $0.5,0.4$ and 0.3 from left to right respectively. Again, at $\gamma=0.3$, the structure breaks down and a series of narrow posts remain.

Each of these were added to the ray-trace model as a deformation matrix and the associated simulated detector image was produced. An example of these are shown in Figure 10. The detector images shown have been highly saturated to show the full effect to the PSF based on the addition of these surface deformations.

The ray-trace model can be used to give the Full Width Half Maximum (FWHM) and Half Energy Width (HEW) of the PSF. The first deformation matrices incorporated in to the ray-trace routine, were a support structure based on the arches design that was used for the first set of printed samples from the experimental theme of this project. ${ }^{4}$ The results from these simple structures were then compared to those of the optimised design. Additional models were also simulated including; the polishing pressure and positive and negative thermal effects on the surface. The ray-trace software can be used to calculate the FWHM and HEW of the simulated detected PSF and a full summary of the results obtained are shown in Table 1.

For some reason, the FWHM is not affected by the change in surface features which seems very odd and is still under investigation. The HEW is changing as the surface features alter and so this is currently being used as a measure of which support structure gives the best results.

The best HEW results of the optimised data sets are obtained from Optimise 1_2 and Optimise 2_1 which both have a $\gamma=0.5$, which is to be expected as this represents the minimum mass removed. Strangely, Optimise $2 \_2$ with a $\gamma=0.4$ has a worse HEW than Optimise $1 \_3$ and Optimise $2 \_3$ which both have a $\gamma=0.3$. Analysis of all results is on-going. However, the HEW would need to be drastically improved from arc minutes to arc seconds to reach the resolution of Chandra and there may potentially be a limit on the resolution that can be obtained via AM. 

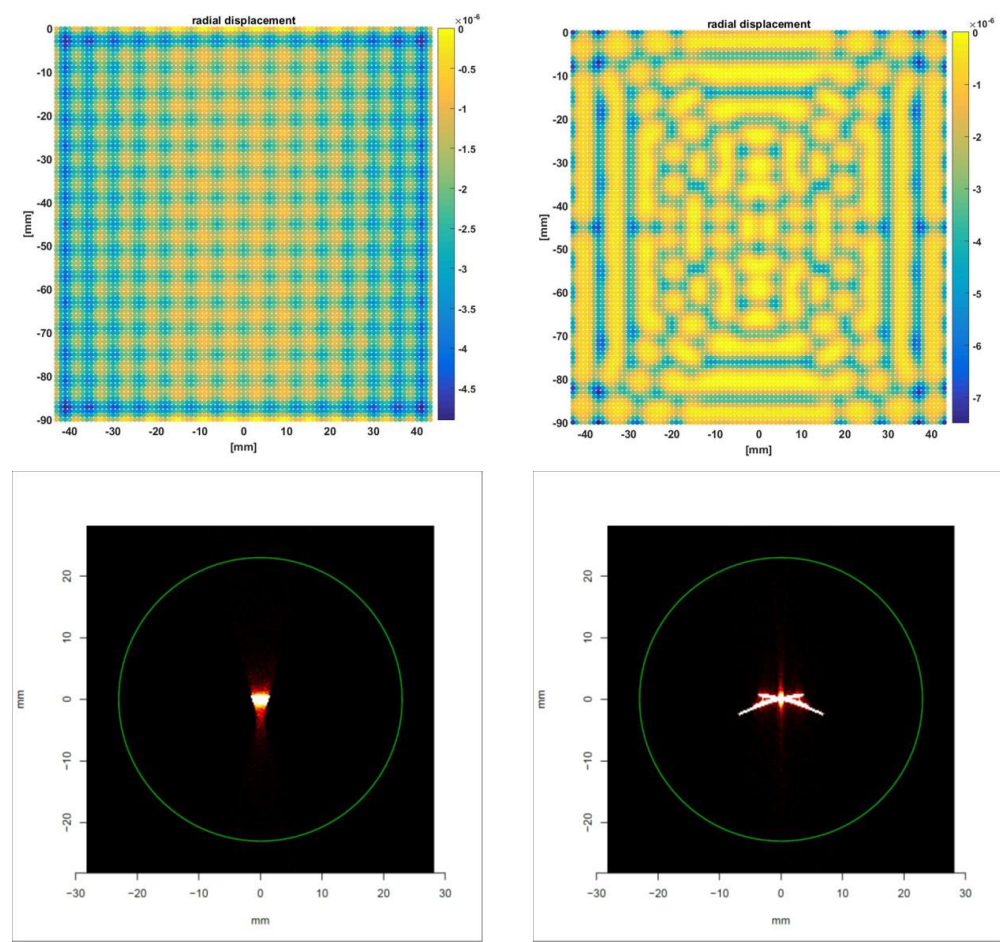

Figure 10. Examples of the deformation matrices which were added to the ray-trace model and the corresponding simulated detector image. Left: The simple arches support structure. ${ }^{4}$ Right: Optimise $1 \_1$ as shown on the left hand side of Figure 8 .

\begin{tabular}{|c|c|c|c|c|}
\hline & FWHM (arcsec) & $\mathrm{HEW}$ (arcmin) \\
\hline \multicolumn{3}{|r|}{ No deformations } & 23.4 & 0.5 \\
\hline \multirow{4}{*}{$\begin{array}{l}\frac{0}{2} \\
\frac{0}{0} \\
\dot{z}\end{array}$} & 1 & ve $\mathrm{Z}$ direction of $3500 \mathrm{~N} / \mathrm{m}^{2}$ & 25.5 & 0.7 \\
\hline & 2 & radial direction of $3500 \mathrm{~N} / \mathrm{m}^{2}$ & 23.0 & 1.8 \\
\hline & 3 & $+10^{\circ} \mathrm{K}$ & 25.0 & 3.7 \\
\hline & 4 & $-10^{\circ} \mathrm{K}$ & 25.0 & 3.5 \\
\hline \multirow{6}{*}{ 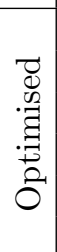 } & $1 \_1$ & $\gamma=0.4$ & 24.5 & 2.2 \\
\hline & 1_2 & $\gamma=0.5$ & 24.8 & 1.0 \\
\hline & $1 \_3$ & $\gamma=0.3$ & 22.7 & 3.4 \\
\hline & $2 \_1$ & $\gamma=0.5$ & 25.0 & 1.1 \\
\hline & $2 \_2$ & $\gamma=0.4$ & 25.7 & 4.4 \\
\hline & $2 \_3$ & $\gamma=0.3$ & 26.9 & 2.7 \\
\hline
\end{tabular}

Table 1. Tabulated results of the FWHM and HEW of the simulated detector images obtained from each of the ray-trace models. 


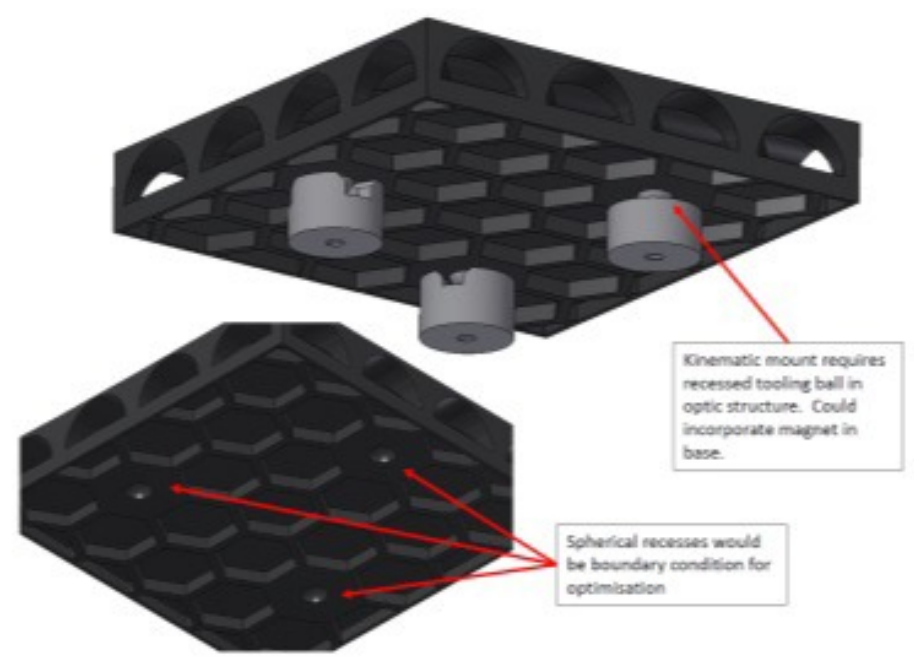

Figure 11. A possible design for the prototype optic which includes the connections to interface with the kinematic mounting scheme shown in Figure 3.
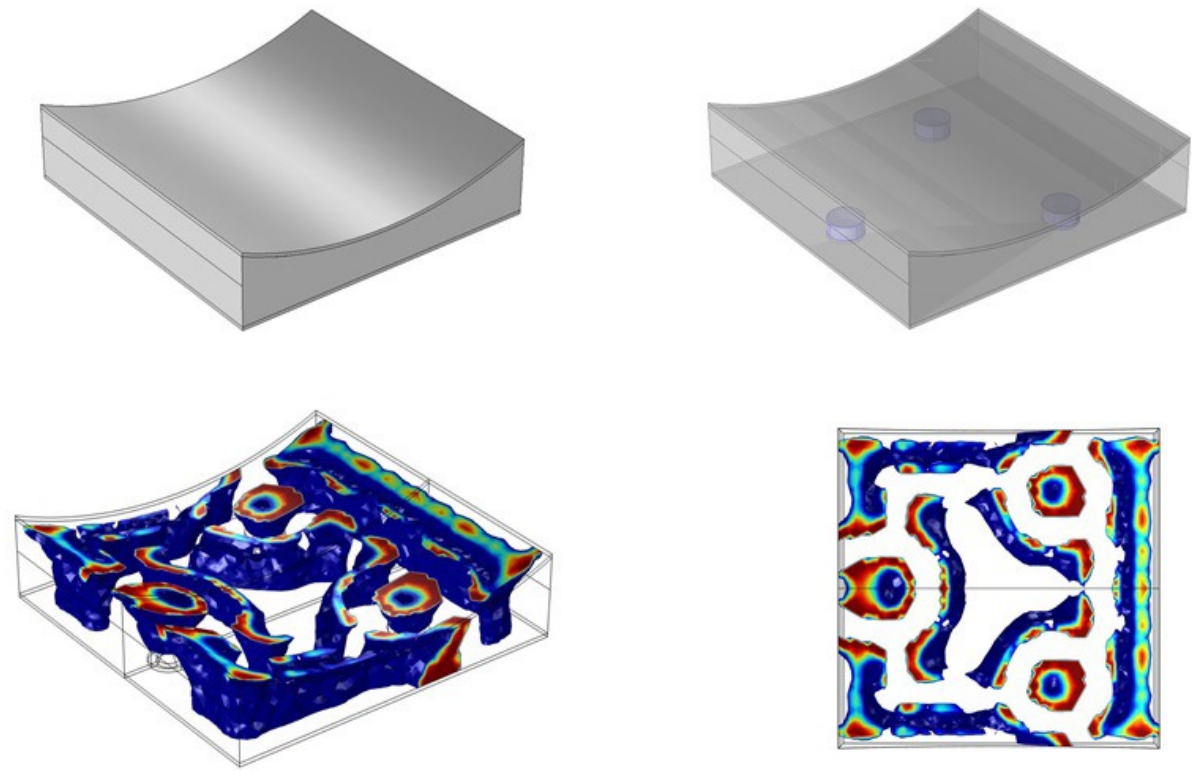

Figure 12. Initial optimised topology of a possible support structure for the design shown in Figure 11 with $\gamma=0.5$.

\section{FUTURE WORK}

As shown in Figure 3, the prototype optic will be supported within the TTF by a kinematic mount. It has been proposed to have the fixtures for the kinematic mount included within the design of the prototype so that they are printed as part of the prototype. An example of such a design is shown in Figure 11.

Initial optimised topology has been completed on this design and the possible underlying support structure with $\gamma=0.5$ has been created. These are shown in Figure 12 but have not yet been modelled using the ray-trace software. Additional models with varying values of $\gamma$ are also being investigated.

\section{SUMMARY AND CONCLUSION}

The work described here is the theoretical theme of a 6 month UK Space Agency's National Space Technology Programme (March 2017 - September 2017), investigating the application of additive manufacturing (AM) 
towards space-based optics. The experimental theme is described in C. Atkins et al., ${ }^{4}$ also presented at this conference. Using the information obtained from the experimental theme, it is hoped that an AM X-ray prototype can be produced which would be tested within the TTF at the University of Leicester. This AM prototype would incorporate an FEA optimised topology support structure which would have been selected using results from the ray-trace software. The aim of this work is to investigate the possibility of producing an X-ray optic with an angular resolution of Chandra at a fraction of the weight.

In the experimental theme, several samples of different materials have been 3D-printed with a simple "arch" support structure and metrology has been completed on pre and post polished samples. A second set of samples have been produced in two materials which have been selected for further investigation as possibilities for the prototype material. These also include an optimised topological support structure. An initial prototype design has been produced to be tested at Leicester and has been ray-trace to give a base line.

FEA models of different optimised support structures have been produced and a "theoretical loop" has been created combining ray-tracing, FEA models and metrology data to predict the optical response. Further optimisation is being completed for the prototype design, which includes the fixtures for the kinematic mounting scheme, with varying values of $\gamma$. Further analysis is required on the current results and the new optimised design needs to be assessed.

\section{ACKNOWLEDGEMENTS}

The authors would like to thank the UK Space Agency for funding this research through the NSTP-3 Pathfinder grant. The authors would also like to thank the metrology team at Glyndŵr Innovations for the use of their Talyor Hobson Form Talysurf Intra in the measurement of the different samples and the staff at CA Models for the help and advice received during the production of the samples. M. Roulet's internship was funded under the H2020 program ERC-STG ICARUS 678777.

This research used the SPECTRE High Performance Computing Facility at the University of Leicester.

\section{REFERENCES}

[1] J. Nella, C. Atkinson, A. Bronowicki, E. Bujanda, A. Cohen, D. Davies, M. Mohan, J. Pohner, P. Reynolds, S. Texter, D. Fitzgerald Simmons, D. Waldie, R. Woods, R. Lynch, R. Lundquist, M. Menzel, B. Smith, P. Sullivan, P. Archeson, and P. Lightsey, "James webb space telescope (jwst) observatory architecture and performance," Proc. of SPIE 5487, 2004.

[2] T. Horn and O. Harrysson, "Overview of current additive manufacturing technologies and selected applications," Science progress 95(3), 2012.

[3] B. Lyons, "Additive manufacturing in aerospace: Examples and research outlook," The Bridge (3), 2014.

[4] C. Atkins, C. Feldman, D. Brooks, S. Watson, W. Cochrane, M. Roulet, P. Doel, R. Willingale, and E. Hugot, "Additive manufactured x-ray optics for astronomy," Proc. of SPIE 10399-52, 2017.

[5] P. Doel, R. Willingale, T. Button, A. James, A. Michette, and T. Stevenson, "Proof of concept, research on smart x-ray optics," submitted to EPSRC EP/D04880X/1, 2005.

[6] C. Atkins, H. Wang, P. Deol, S. Thompson, D. Brooks, C. Feldman, R. Willingale, T. Button, D. Zhang, D. Rodriguez Sanmartin, A. James, and C. Theobald, "Active x-ray optics for the next generation of x-ray telescopes," Proc. of SPIE 7360, p. 736008, 2009.

[7] C. Feldman, R. Willingale, C. Atkins, H. Wang, P. Deol, S. Thompson, D. Brooks, T. Button, D. Zhang, D. Rodriguez Sanmartin, A. James, and C. Theobald, "Development of thin shell adaptive optics for high angular resolution x-ray telescopes," Proc. of SPIE 7011, p. 70110Y, 2008.

[8] COMSOL: COMSOL Multiphysics Modeling and Simulation, "http://www.comsol.com/." 